\title{
CLINICAL ELECTRORETINOGRAPHY. I
}

The human eye generates an electrical potential of about 6 millivolts which may vary by several millivolts over a period of minutes. This potential has been named the "steady potential" to distinguish it from the more rapid variation that occurs when the eye is exposed to light. The rapid potential change is very fast, being completed in less than half a second, and is relatively small, rarely exceeding 0.5 millivolt $(500$ microvolts, $\mu \mathrm{v})$. This rapid potential change is the electroretinogram (ERG).

The ERG is a polyphasic wave (a wave comprising several peaks and troughs) which shows wide species variation, but the clinical ERG is relatively simple, comprising an initial negative deflection ( $a$-wave) followed by a larger positive deflection ( $b$-wave) (Fig. 1). Each wave may be subdivided into an early photopic component $\left(a_{p}\right.$ and $\left.b_{p}\right)$ and a later scotopic component $\left(a_{s}\right.$ and $\left.b_{s}\right)$. The $b_{p}$ wave is sometimes known as the $x$-wave, and under certain circumstances wavelets may be seen on the ascending limb of the $b$-wave, the oscillatory potentials (or $e$-waves). Other waves have been described: the $c$-wave is a later positive response, and the $d$-wave a sudden fall in potential at the end of the response, but these are not evident in the usual clinical ERG.

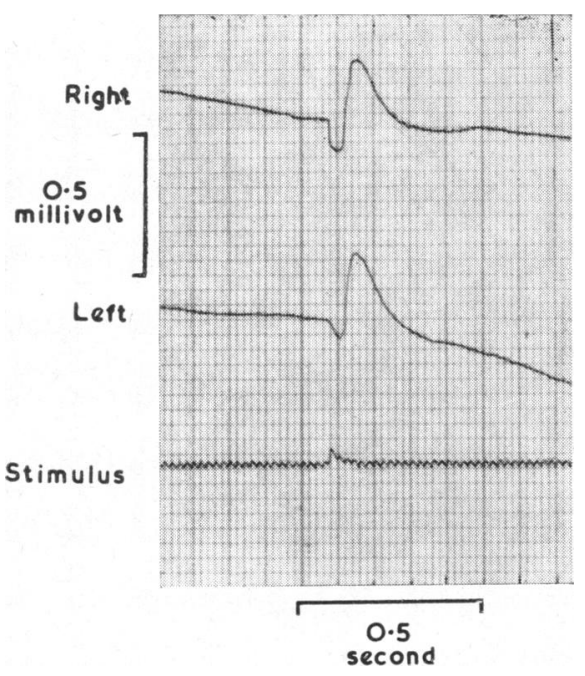

Fig. 1.-Normal ERG.

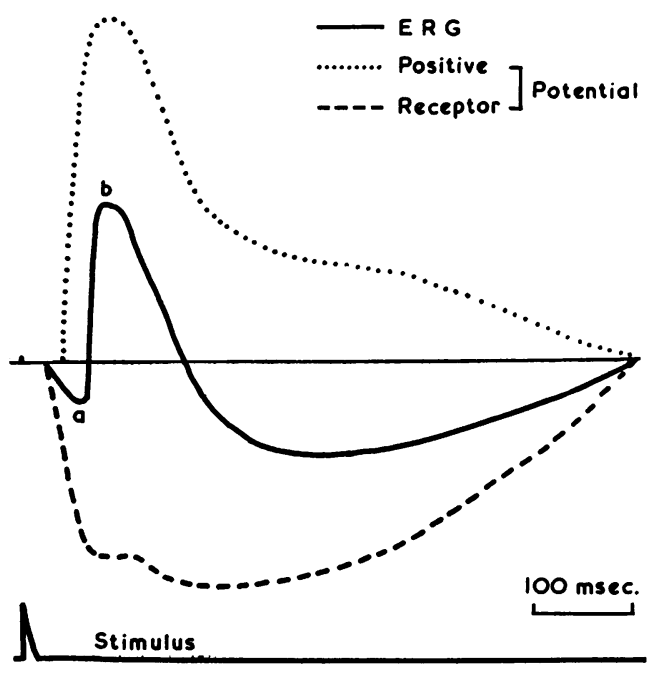

FIG. 2.-Production of ERG waveform.

\section{Physiological Basis}

The ERG waveform is produced by the interaction of two processes (Fig. 2):

(1) Receptor Potential (Granit's P III).-This is an early negative phase, the leading edge of which forms the $a$-wave. Recently this has been subdivided into an early and a late component. It arises in the receptor layer.

(2) Positive Potential (Granit's P II).- - This arises in the inner nuclear layer and is positive. It is later than the receptor potential but generally larger and produces the $b$-wave.

The origin of the oscillatory potentials is unknown. 


\section{Measurement of the ERG}

There are many ways of describing the ERG, but the usual methods are as follows:

(1) Height of Wave.- $a$-wave measured from the baseline, $b$-wave measured from the apex of the $a$-wave (but by some workers from the baseline).

(2) Latency.-This is the time taken for an event to start after the stimulus.

(3) Culmination (Implicit) Time.-This is the time taken for an event to reach its maximal height after the stimulus.

(4) Duration of the Response.

These few variables can give an almost infinite variation in the ERG waveform which is controlled by a large number of factors.

\section{Factors influencing the Waveform of the ERG}

(1) The Stimulus

(a) Intensity. The $a$-wave increases more than the $b$-wave at high intensities.

(b) Duration.

(c) Spectral composition. The scotopic elements are relatively insensitive to the long wavelengths.

(d) Area.

(e) Repetition. Rapidly repeated stimuli give a smaller second response, while very rapid stimulation (flicker) leads to fusion of the response and a smooth record at the "fusion frequency".

(2) The Apparatus

(a) Electrode position.

(b) Amplifier. The various electronic constants incorporated in this modify the waveform.

(c) Display method. A cathode ray oscilloscope will record the amplifier output faithfully, but for convenience a direct writing machine is generally used with its concomitant mechanical deficiencies.

(3) The Eye

(a) State of dark adaptation. As dark adaptation proceeds the ERG increases in size and the scotopic elements predominate.

(b) Pupil size. A large pupil allows more light to enter the eye. If a cycloplegic has been used the record does not contain electrical responses produced by the iris and ciliary muscle in response to light.

(c) Age and sex. Except in the premature or very young baby these factors are unimportant.

(d) Eye disease. ERG changes occur in many diseases and systemic disorders, and it is the existence of these that form the basis of clinical electroretinography.

(To be concluded)

JOHN H. KeLSEY,

Electrodiagnostic Department, MOORFIELDS EYE HosPITAL, LONDON, E.C.1. 